\title{
2.5 GBPS 2-PSK ULTRA-DENSE WDM HOMODYNE COHERENT DETECTION USING A SUB-CARRIER BASED OPTICAL PHASE-LOCKED LOOP
}

\author{
S. Camatel, V. Ferrero, R. Gaudino and P. Poggiolini \\ PhotonLab, Dipartimento di Elettronica, Politecnico di Torino, Corso Duca degli Abruzzi 24, \\ 10129 Torino, Italy
}

\begin{abstract}
We present an optical phase locked loop based on sub-carrier modulation and designed using commercial optical components. In principle the output of a continuous-wave laser is subcarrier modulated by a common LiNbO3 MachZehender driven by an electrical voltage controlled oscillator. The proposed architecture is used as optical receiver for homodyne coherent detection of ultra-dense WDM 2.5 Gbps 2-PSK signals with $6.25 \mathrm{GHz}$ spacing. This method offers the potential for providing many closely spaced multigigabit channels and enables coherent lightwave technology to become commercially viable.
\end{abstract}

\section{INTRODUCTION}

The development of Ultra-Dense WDM (UDWDM) systems is currently under investigation for increasing the global capacity per single fiber. For example in [1], $10 \mathrm{Gbit} / \mathrm{s}$ transmission at $25 \mathrm{GHz}$ channel spacing is demonstrated and studied in details. Anyway, it is shown in this and other works that optical filters for UDWDM have very tight requirements in pass-band shape and frequency stability. As described in [2] these requirements can be avoided using coherent communication systems which let transmission of a large number of WDM optical channels with very narrow frequency separations. Another advantage of coherent transmission is the ability to select any particular channel by simply tuning a local oscillator. An optical phase-locked loop (OPLL) has then to be implemented in order to obtain a tunable local oscillator.

OPLLs for homodyne or heterodyne coherent detection received a great deal of attention at the beginning of the 1990s [3] in order to increase the receiver sensitivity, but they never found practical applications, first because of their 
complexity, secondly due to the introduction of EDFAs, that greatly leveraged sensitivity issues. We proposed in [4-6] a novel and much simpler OPLL architecture based on commercial off-the-shelf optoelectronic components and without fast direct laser frequency tuning. Frequency tuning is obtained in our system through optical sub-carrier generation and tuning, and will be indicated as SC-OPLL.

This paper presents a possible application of our SC-OPLL for demultiplexing and detection of UDWDM signals. We propose and demonstrate a receiver setup capable of detecting 2-PSK signals at $2.5 \mathrm{Gbit} / \mathrm{s}$ and $6.25 \mathrm{GHz}$ channel separation. Our setup does not require narrow optical filters, and can be an enabling technology for future optical networks.

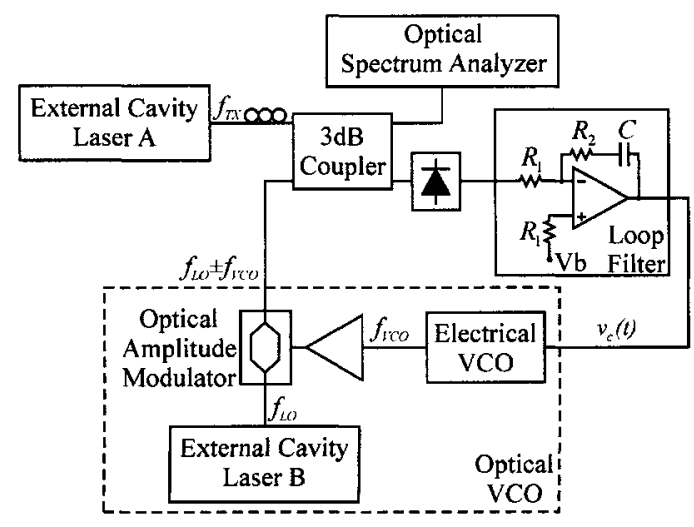

Figure 1. Schematic diagram of SC-OPLL.

\section{SC-OPLL ARCHITECTURE}

The SC-OPLL architecture is shown in Fig. 1. The Optical Voltage Controlled Oscillator (OVCO) is the key-element and is based on a commercial Continuous-Wave (CW) external cavity tunable laser at frequency $f_{L O}$ that is externally amplitude modulated by the signal coming from an electrical VCO at frequency $f_{V C O}$. By biasing the external Mach-Zehnder (MZ) amplitude modulator at a null of its transfer function, a sinusoidal carrier-suppressed modulation is obtained. The resulting spectrum at the output of the OVCO is shown in Fig. 2. Two main sub-carriers at frequency $f_{L O} \pm f_{V C O}$ are generated, with spurious optical tones at $f_{L O}$ and $f_{L O} \pm 2 \cdot f_{V C O}$ due respectively to a limited extinction ratio and nonlinearities of the amplitude modulator. Considering one of the two main sub-carriers, for example the one at $f_{L O}+f_{V C O}$, we are able to tune an optical frequency by simply changing the voltage applied to the electrical VCO, thus implementing an OVCO. Actually, this is the key issue of 


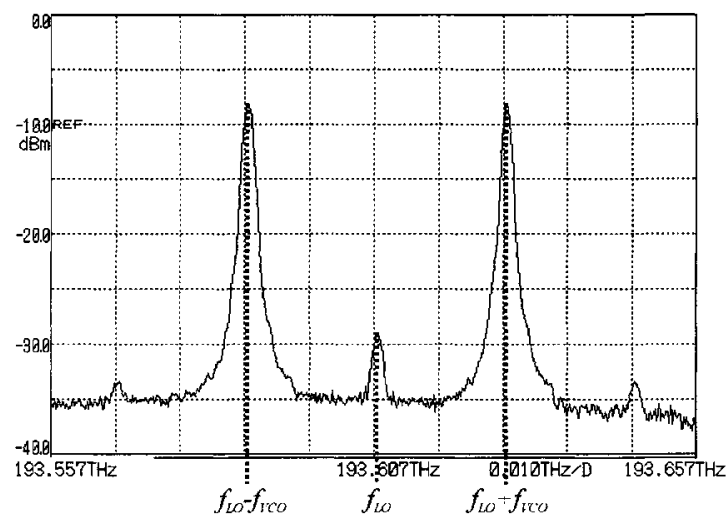

Figure 2. Spectrum at the output of the optical VCO.

the proposed architecture, that allows obtaining optical fine frequency tuning with the speed and stability of an electrical VCO, and thus to re-use typical RF PLL set-ups. In our case, a standard second-order PLL control circuit allows to lock $f_{L O}+f_{V C O}$ to the transmitted signal at frequency $f_{T X}$ (laser A in Fig. 1). When the OPLL is locked to $f_{L O}+f_{V C O}=f_{T X}$, optical homodyne is obtained, allowing to track the incoming optical signal frequency and phase.

When the incoming signal is modulated, its optical spectrum is translated to base-band at the photodiode output. In principle, due to beating with the other sub-carriers, copies of this signal appears also around frequencies $f_{V C O}$ and $2 \cdot f_{V C O}$, but they can be filtered out by the receiver filter if $f_{V C O}$ is larger than the signal spectral width (or bandwidth). Other details of the setup can be found in Fig. 1. A polarization controller matches the polarization of TX and LO signals before being combined by a $3 \mathrm{~dB}$ coupler and sent to an amplified photodiode. The resulting electrical signal is processed by a single-pole active filter, in order to obtain a second-order PLL with natural frequency $f_{\text {loop }}$ and the damping factor $\xi$ [5], which both depend on the loop filter parameters $\tau_{1}=\mathbf{R}_{1} \mathrm{C}$ and $\tau_{2}=\mathrm{R}_{2} \mathrm{C}$.

\section{ULTRA-DENSE WDM COHERENT DETECTION}

An ultra-dense WDM optical transmission system has been experimentally set up in order to evaluate the performance of our SC-OPLL. The description of the system's design and the obtained results follow.

\subsection{System setup}

The system experimental setup is shown in Fig. 3, and is an UDWDM extension of the one presented in [4]. Three $\mathrm{CW}$ lasers at frequency $f_{0}$ (central 


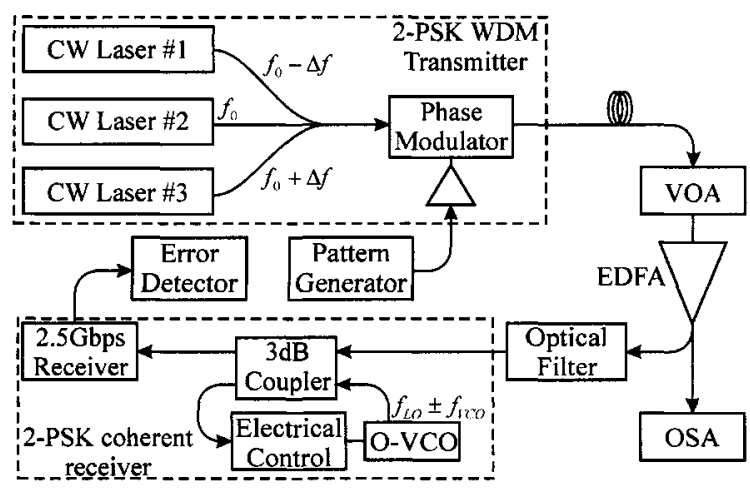

Figure 3. UD-WDM experimental setup.

channel) and $f_{0} \pm \Delta f$ (adjacent channels) were optically multiplexed and sent to a $\mathrm{LiNbO}_{3}$ external phase modulator, driven by an electrical NRZ $2.5 \mathrm{Gbit} / \mathrm{s}$ PRBS signal to obtain a 2-PSK modulation. We used external cavity tunable lasers in order to be able to freely set the UDWDM channel separation $\Delta \mathrm{f}$. The resulting optical signal, whose spectrum is shown in the upper part of Fig. 4, is sent to a variable optical attenuator (VOA) and then to an optical preamplifier. The resulting signal is then filtered by a $0.6 \mathrm{~nm}$ optical filter, which is used to reduce ASE noise, but is large enough to let the three channels pass through undistorted, i.e. this filter does not perform WDM demodulation. The coherent receiver is based on our SC-OPLL. The local oscillator frequency $f_{L O}$ has been

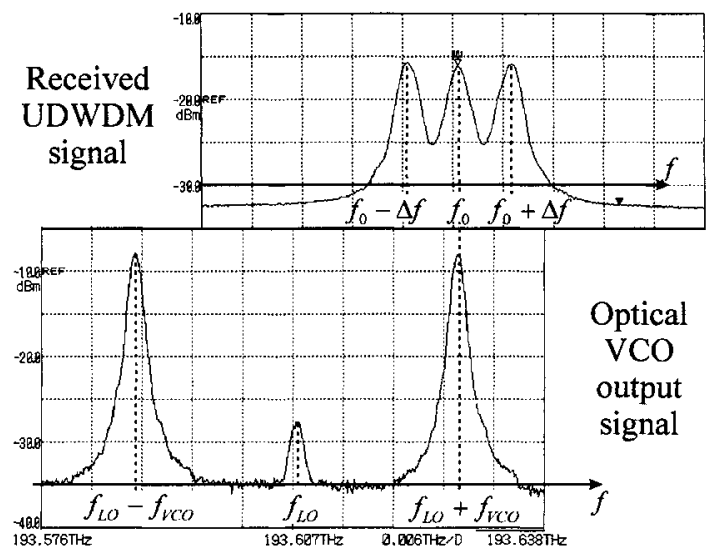

Figure 4. Spectra ( $0.01 \mathrm{~nm}$ resolution bandwidth) of the received UD-WDM signal (a) and of the OVCO output. 
set such that the main sub-carrier $f_{L O}+f_{V C O}$ is able to lock the transmitted central channel frequency $f_{0}$. The spectrum at the output of the OVCO has been represented in the lower part of Fig. 4 which graphically represents the desired locking condition. The $2.5 \mathrm{Gbps}$ receiver is a typical amplified photodiode and shifts the received WDM spectrum to base-band. Indeed, when the SC-OPLL is locked, the spectrum at the output of the photodetector includes a spectral component centered on zero frequency generated by the central channel and the contribution around $\Delta f$ given by the adjacent two channels. UD-WDM channel demultiplexing and demodulation is directly obtained through the receiver electrical filters. In fact, a standard receiver filter such as SDH 4-poles Bessel filter at $1.8 \mathrm{GHz}$ proved more than adequate to reject the two adjacent channels efficiently. In contrast, a standard DWDM receiver would demultiplex through optical filtering which is quite impractical at $6.25 \mathrm{GHz}$ channel spacing.

In our experiment, we used a 2-PSK modulation with residual carrier, so that the SC-OPLL automatically locks to the 2-PSK residual carrier. For further details on this principle see [8]. The O-VCO contains an electrical VCO with $f_{V C O}=20 \mathrm{GHz}$ and $5 \mathrm{GHz}$ dynamic range, followed by a $40 \mathrm{GHz}$ bandwidth $\mathrm{LiNbO}_{3}$ amplitude modulator.

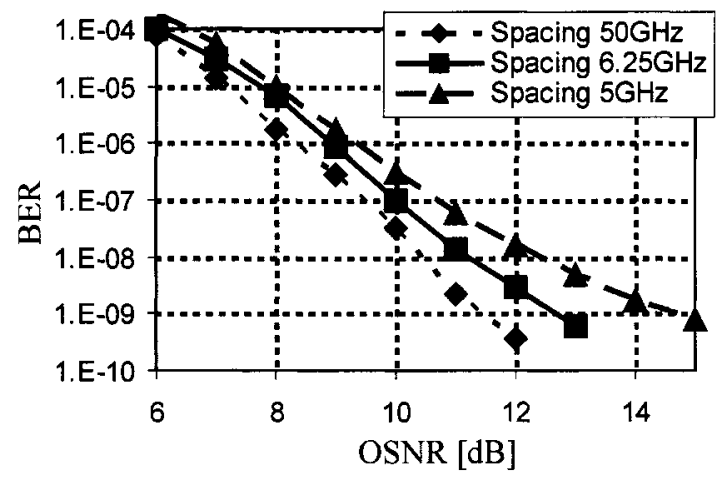

Figure 5. BER vs. received OSNR (0.1 nm resolution bandwidth) for $50,6.25$ and $5 \mathrm{GHz}$ channel spacing values.

\subsection{Experimental Results}

The performances of our setup were tested by measuring the BER as a function of the OSNR (measured over $0.1 \mathrm{~nm}$ resolution) for different channel spacing. The results are shown in Fig. 5 for $\Delta f$ equal to $50,6.25$ and $5 \mathrm{GHz}$. The curve for $\Delta f=50 \mathrm{GHz}$ can be taken as a reference for a situation without WDM crosstalk. In fact, we verified that it does not show any penalty with 
respect to single channel transmission. The curve for $\Delta f=6.25 \mathrm{GHz}$ shows a penalty smaller than $1 \mathrm{~dB}$ at $\mathrm{BER}=10^{-9}$. This result proves the feasibility of the proposed setup, even for $6.25 \mathrm{GHz}$ channel spacing. The penalty becomes larger (more than $3 \mathrm{~dB}$ ) for $\Delta f=5 \mathrm{GHz}$, where the UD-WDM channel spectra significantly overlap, thus giving rise to an intrinsic, receiver independent, channel crosstalk. Please note that we do not use any particular signal shaping at the transmitter [7], so that $\Delta f=5 \mathrm{GHz}$, i.e., twice the bit rate, is close to the theoretical limits (regarding bandwidth occupation).

In our experiment, due to hardware limitation, we used only three wavelengths at the transmitter side, which are anyway sufficient as a "proof-ofconcept" of our technique. In a practical setup with many UD-WDM channels, when $f_{L O}+f_{V C O}=f_{O}$, the other SC-OPLL subcarrier at $f_{L O}-f_{V C O}$ could beat with another WDM channel. Anyway, this problem can be easily solved by using an optical filter with a passband of the order of $2 f_{V C O}$, which could be significantly greater than $\Delta f$. In our experiment, we had $20 \mathrm{GHz}$, thus envisioning the use of a quite common $40 \mathrm{GHz}$ bandwidth optical filter (tunable, if required by the network architecture).

\section{CONCLUSIONS}

We proposed a novel optical PLL architecture based on off-the-shelf optoelectronic components and we used it for coherent detection of $2.5 \mathrm{Gbit} / \mathrm{s}$ 2-PSK signals with $6.25 \mathrm{GHz}$ spacing and $1 \mathrm{~dB}$ penalty for UDWDM applications. The use of optical homodyning, greatly mitigates the requirements on optical filtering and enables channel spacing in the few $\mathrm{GHz}$ range. The price to be paid for optical homodyning is the receiver complexity. Anyway, most of the components required in our setup could potentially be integrated using next-generation optical circuits and devices, thus opening new possibilities for future optical transmission systems, and allowing at the same time a reduction in the costs.

\section{ACKNOWLEDGMENTS}

This project was partially funded by CISCO, University Research Program (URP). The authors would like to thanks S. Morasca (Avanex) for his invaluable support to the experiment.

\section{REFERENCES}

[1] I. Lyubomirsky, T. Qui, J. Roman, M. Nayfeh, M. Y. Frankel and M. G. Taylor, "Interplay of Fiber Nonlinearity and Optical Filtering in Ultradense WDM," IEEE Photonics Technology Letters, vol. 15, no. 1, pp. 147-149, Jan. 2003.

[2] R. E. Wagner, N. K. Cheung and P. Kaiser, "Coherent Lightwave Systems for Interoffice and Loop-Feeder Applications," Journal of Lightwave Technology, vol. LT-5, no. 4, April 1987. 
[3] L. G. Kazovsky and D. A. Atlas, "A 1320-nm experimental optical phase-locked loop: performance investigation and PSK homodyne experiments at $140 \mathrm{Mbps}$ and $2 \mathrm{Gbps",}$ Journal of Lightwave Technology, vol. 8, no. 9, pp. 1414-1425, Sept. 1990.

[4] S. Camatel, V. Ferrero, R. Gaudino and P. Poggiolini, "10 Gbit/s 2-PSK Transmission and Homodyne Coherent Detection using Commercial Optical Components," ECOC 2003, paper We.P.122.

[5] V. Ferrero, S. Camatel, R. Gaudino and P. Poggiolini, "A novel Optical Phase Locked Loop architecture based on Sub-Carrier modulation," OFC 2004, paper FN6.

[6] S. Camatel, V. Ferrero, R. Gaudino and P. Poggiolini, "Optical phase-locked loop for coherent detection optical receiver," IEE Electronics Letters, vol. 40, no. 6, pp. 384-385, March 2004.

[7] L. G. Kazovsky, S. Benedetto and A. Willner, Optical fiber communication systems, chap. 4, Artech House, 1996.

[8] L. Kazovsky, "Balanced Phase-Locked Loops for Optical Homodyne Receivers: Performance Analysis, Design Considerations, and Laser Linewidth Requirements," Journal of Lightwave Technology, vol. LT-4, no. 2, pp. 182-195, Feb. 1986. 